\title{
The Association between Bone Quality and Atherosclerosis: Results from Two Large Population-Based Studies
}

\author{
V. Lange, ${ }^{1}$ M. Dörr, ${ }^{2,3}$ U. Schminke, ${ }^{4}$ H. Völzke, ${ }^{3,5}$ M. Nauck, ${ }^{1,3}$ H. Wallaschofski, ${ }^{1,3}$ and \\ A. Hannemann ${ }^{1}$ \\ ${ }^{1}$ Institute of Clinical Chemistry and Laboratory Medicine, University Medicine Greifswald, Greifswald, Germany \\ ${ }^{2}$ Department for Internal Medicine B, University Medicine Greifswald, Greifswald, Germany \\ ${ }^{3}$ German Centre for Cardiovascular Research, Partner Site Greifswald, Greifswald, Germany \\ ${ }^{4}$ Department for Neurology, University Medicine Greifswald, Greifswald, Germany \\ ${ }^{5}$ Institute for Community Medicine, University Medicine Greifswald, Greifswald, Germany
}

Correspondence should be addressed to A. Hannemann; anke.hannemann@uni-greifswald.de

Received 20 April 2017; Accepted 3 July 2017; Published 9 August 2017

Academic Editor: Astrid Fahrleitner-Pammer

Copyright (c) 2017 V. Lange et al. This is an open access article distributed under the Creative Commons Attribution License, which permits unrestricted use, distribution, and reproduction in any medium, provided the original work is properly cited.

\begin{abstract}
Objective. It is highly debated whether associations between osteoporosis and atherosclerosis are independent of cardiovascular risk factors. We aimed to explore the associations between quantitative ultrasound (QUS) parameters at the heel with the carotid artery intima-media thickness (IMT), the presence of carotid artery plaques, and the ankle-brachial index (ABI). Methods. The study population comprised 5680 men and women aged 20-93 years from two population-based cohort studies: Study of Health in Pomerania (SHIP) and SHIP-Trend. QUS measurements were performed at the heel. The extracranial carotid arteries were examined with B-mode ultrasonography. ABI was measured in a subgroup of 3853 participants. Analyses of variance and linear and logistic regression models were calculated and adjusted for major cardiovascular risk factors. Results. Men but not women had significantly increased odds for carotid artery plaques with decreasing QUS parameters independent of diabetes mellitus, dyslipidemia, and hypertension. Beyond this, the QUS parameters were not significantly associated with IMT or ABI in fully adjusted models. Conclusions. Our data argue against an independent role of bone metabolism in atherosclerotic changes in women. Yet, in men, associations with advanced atherosclerosis, exist. Thus, men presenting with clinical signs of osteoporosis may be at increased risk for atherosclerotic disease.
\end{abstract}

\section{Introduction}

Osteoporosis and atherosclerosis substantially impact the elderly, leading to increased morbidity and mortality $[1,2]$. Osteoporosis, on the one side, is a chronic disease characterized by low bone mass, microarchitectural deterioration of bone tissue, and an increased fracture risk [1]. Based on these diagnostic criteria, 27.6 million women and men in the European Union are affected by osteoporosis [1]. Atherosclerosis, on the other side, is characterized by loss of elasticity of the artery walls, wall thickening, and plaque formation [2]. Cardiovascular disease due to atherosclerosis represents a major economic burden on the European health care system, with estimated annual costs of 192 billion euros [3]. Moreover, cardiovascular disease is the leading cause of death worldwide, with more than 17.5 million deaths in 2012 [4].

Osteoporosis and atherosclerosis share common risk factors like aging, dyslipidemia, oxidative stress, inflammation, hypertension, and diabetes $[2,5]$. Furthermore, they share molecular pathways involving, for example, bone and vascular mineralization or inflammatory processes [6]. Despite these common risk factors, epidemiologic studies suggested an independent association between low bone mass and atherosclerosis $[5,7]$. Thus, a population-based study including [5] 2726 postmenopausal women and 2543 men from 
Norway reported associations between low bone mineral density (BMD) and echogenic calcified atherosclerotic plaques. Further, lower volumetric trabecular lumbar BMD was associated with advanced carotid plaque in 1833 postmenopausal women and men from a subsample of the Multi-Ethnic Study of Atherosclerosis [7]. In that study [7], also, associations of lower volumetric trabecular lumbar $\mathrm{BMD}$ with lower ankle-brachial index $(\mathrm{ABI})$ and increased internal carotid artery intima-media thickness (IMT) were found in men but not in women. A male-specific inverse association between femoral neck BMD and the 10-year risk for coronary heart disease was also described in a study including 5415 men and 7409 women from the general population in Korea [8]. On the other side, associations between BMD and pulse wave velocity [9] as well as between BMD loss and coronary artery calcification [10] were described for women only. The previous study results [5, 7-10] appear even more controversial when considering that competing studies reported no associations between a low BMD and carotid IMT as well as pulse wave velocity and ABI [11] or that associations between peripheral artery disease and bone loss turned nonsignificant after age adjustment [12].

Taken together, it is still uncertain whether low bone quality is associated with atherosclerotic changes independent of major cardiovascular risk factors like diabetes mellitus, dyslipidemia, or hypertension or whether sexspecific differences exist. Besides, there are only few data in the association between bone quality at the heel, measured by quantitative ultrasound (QUS), and atherosclerosis $[13,14]$. We therefore aimed to explore the associations between QUS-based parameters, with carotid artery IMT, the presence of carotid artery plaques, and the ABI in the general population.

\section{Subjects and Methods}

2.1. Study Populations. The present analysis is based on data from two population-based cohort studies in northeast Germany: the second follow-up of the Study of Health in Pomerania (SHIP-2) and SHIP-Trend. Details on the study design, protocols, and sampling methods have been reported elsewhere $[15,16]$. In short, the baseline examinations in the SHIP cohort were performed between 1997 and 2001 with a total of 4308 men and women aged 20-81 years. The second follow-up (SHIP-2) was performed between 2008 and 2012 with 2333 subjects aged 30-93 years being re-examined. The baseline examinations in the SHIP-Trend cohort were performed in parallel with SHIP-2 between 2008 and 2012 with a total of 4420 adult men and women aged $20-84$ years. All investigations were carried out in accordance with the Declaration of Helsinki, including written informed consent of all participants. The study methods were approved by an institutional review board (ethics committee at the University of Greifswald).

Data from SHIP-2 and SHIP-Trend were pooled for the present analyses. From the resulting population of $N=6753$, all subjects with missing data in QUS, IMT, plaque, or confounder variables were excluded as well as subjects with extremely high values in the QUS variables, pregnant women, subjects with estimated glomerular filtration rate (eGFR) below $30 \mathrm{ml} / \mathrm{min} / 1.73 \mathrm{~m}^{2}$ or missing eGFR, and those who reported with intake of bisphosphonates, selective estrogen receptor modulators, parathyroid hormone, steroids, or strontium ranelate (for details, see Supplemental Figure 1 available online at https://doi.org/10.1155/2017/ 3946569). The resulting study population comprised 5680 subjects. Among these subjects, two-thirds (67.8\%) participated in the ABI examination, resulting in a subsample of 3853 subjects.

2.2. Interview and Physical Examination. All SHIP-2 and SHIP-Trend participants were offered a large range of standardized medical examinations, biomaterial sampling, and an extensive computer-aided personal interview. During the personal interview, information on sociodemographic characteristics, lifestyle, and medical histories were collected. All participants were asked to bring their medications taken seven days prior to the time of examination. Medication data were obtained online using the IDOM program (online drugdatabase leaded medication assessment) and classified using the Anatomical-Therapeutic-Chemical (ATC) classification system. Intake of bisphosphonates was defined as ATC M05BA-BB, selective estrogen receptor modulators as ATC G03XC, PTH preparations as ATC H05AA, strontium ranelate as ATC M05BX03, glucocorticoids for systemic use as ATC $\mathrm{H} 02 \mathrm{AB}$ and $\mathrm{H} 02 \mathrm{BX}$, vitamin D preparations as ATC A11CC, and cardioprotective medication as ATC C. Participants were defined as physically inactive if they reported less than one hour of regular physical activity per week during summer and winter. Risky alcohol consumption was defined as alcohol intake at or above $30 \mathrm{~g} /$ day in men and $20 \mathrm{~g} /$ day in women. All women older than 60 years of age and women between 40 and 60 years of age without self-reported menstrual cycling were classified as postmenopausal. Years since menopause were calculated as the difference between current age and age at last menstruation. Standardized measurements of body height and weight were performed with calibrated scales. Body mass index (BMI) was calculated as weight $(\mathrm{kg}) /$ height $^{2}\left(\mathrm{~m}^{2}\right)$. Systolic and diastolic blood pressures were measured three times on the right arm of seated subjects, using a digital blood pressure monitor (HEM705CP, Omron Corporation, Tokyo, Japan). The mean of the second and third measurements was used for statistical analyses. Hypertension was defined as systolic blood pressure $\geq 140 \mathrm{mmHg}$ or diastolic blood pressure $\geq 90 \mathrm{mmHg}$ or self-reported intake of antihypertensive medication. Diabetes mellitus was defined when a respective physician's diagnosis or intake of antidiabetic medication (ATC A10) was reported, when $\mathrm{HbA} 1 \mathrm{c}$ was $\geq 6.5 \%$ or serum glucose concentrations were $\geq 11.1 \mathrm{mmol} / \mathrm{l}$. Dyslipidemia was defined as total cholesterol concentration $\geq 6.2 \mathrm{mmol} / \mathrm{l}$ or LDLcholesterol concentration $\geq 4.1 \mathrm{mmol} / \mathrm{l}$ or HDL-cholesterol concentration $<1.04 \mathrm{mmol} / \mathrm{l}$ or triglycerides $\geq 1.7 \mathrm{mmol} / 1$ or intake of lipid-modifying agents (ATC C10).

2.3. QUS. QUS at the heel was performed using the Achilles InSight device (GE Medical Systems Ultrasound, GE Healthcare, Chalfont St. Giles, U.K.), a water-based bone 
ultrasonometer, as reported previously [17]. Two devices without systematic differences were used during the course of the study. The measurements were performed successively on both feet of the seated participants by trained and certified examiners. Alcohol was used as a coupling agent. The system measures the frequency-dependent attenuation of the sound waves (broadband ultrasound attenuation (BUA)) and the speed of sound waves (SOS) as they pass through the heel (os calcis). BUA and SOS were combined to form the stiffness index according to the following formula: $\quad$ stiff fness index $=(0.67 \times \mathrm{BUA})+(0.28 \times \mathrm{SOS})-420$. The system automatically compares individual stiffness index results to values obtained in a healthy young reference population. Indices below the reference mean minus 2.5 standard deviations were taken to indicate a high osteoporotic fracture risk, indices above the reference mean minus 2.5 standard deviations but below the reference mean minus 1 standard deviations were taken to indicate a medium osteoporotic fracture risk, and indices above the reference mean minus 1 standard deviations were taken to indicate a low osteoporotic fracture risk [17].

2.4. IMT and Plaques. Certified medical assistants examined the extracranial carotid arteries with B-mode ultrasonography (vivid-i, GE Medical Systems, Waukesha, WI, USA) using a broad-bandwidth linear array transducer with an operating frequency of $13 \mathrm{MHz}$. Longitudinal scans of the distal straight portion of the far wall of the common carotid artery (CCA) of both sides were recorded. CCA-IMT was assessed on-screen using a semiautomated edge tracking software, which measures the distance between the lumenintima and media-adventitia interfaces at an arterial segment of $1 \mathrm{~cm}$ in length located directly proximal to the widening of the artery at the bifurcation. The "mean CCA-IMT," which was used for statistical analyses, was calculated as the average of the mean values of 250 measurement points of each side. The carotid arteries were further evaluated in longitudinal and cross-sectional scans for the presence of atherosclerotic plaques. Each arterial segment (i.e., the left or right common carotid artery, internal carotid artery, external carotid artery, and the carotid bifurcation) was categorized into either affected by plaque or plaque-free. If at least one arterial segment was classified as being affected by plaque, "carotid plaques" were defined as being present. Further, the number of arterial sites affected by plaque ranging between zero and eight was recorded.

2.5. ABI. The Doppler method was used to determine systolic blood pressure in both arms (brachial artery) and both ankles (anterior and posterior tibial arteries) for the calculation of the ABI. After at least ten minutes of rest in the supine position, the measurements were started. The measurements were performed with the "Dopplex D900" (Huntleigh Healthcare Ltd., Cardiff, U.K.) and a blood pressure cuff (Welch Allyn, Skaneateles Falls, USA). The calculation of the ABI followed the guidelines of the American Heart Association [18]. The higher of the anterior and posterior tibial artery systolic blood pressure of each leg was divided by the higher of the right or left brachial artery systolic blood pressure. The lower of the right or left leg ABIs was used for statistical analyses.

2.6. Laboratory Measurements. Blood samples were taken between 7 a.m. and 1 p.m. from participants in the supine position. Creatinine, glucose, total cholesterol, triglyceride, HDL-cholesterol, and LDL-cholesterol serum concentrations were measured on the Dimension Vista (Siemens Healthcare Diagnostics, Eschborn, Germany). Hbalc concentrations were measured with high performance liquid chromatography on a DIAMAT analyzer (Bio-Rad Laboratories, Munich, Germany). Serum 25-hydroxy vitamin D (25OHD) concentrations were measured in a subgroup of 3540 (62.3\%) SHIP-Trend participants with the IDS-iSYS 25-hydroxy vitamin D assay on the IDS-iSYS Multidiscipline Automated Analyser (Immunodiagnostic Systems Limited, Frankfurt am Main, Germany). The eGFR was calculated according to the 4-variable modification of diet in renal disease equation [19].

2.7. Statistical Analyses. Due to previously reported marked differences between men and women in the association between bone metabolism and atherosclerotic changes, all analyses were stratified by sex. Continuous data are expressed as median (1st-3rd quartiles) and nominal data as percentage. The Kruskal-Wallis test or the $\chi^{2}$ test was used for group comparisons. A value of $p<0.05$ was considered statistically significant.

Analyses of variance (ANOVA) and linear and logistic regression analyses were performed to assess the associations of the QUS-based parameters (exposures: BUA, SOS, stiffness index, and risk for osteoporotic fractures) with the cardiovascular parameters (outcomes: IMT, carotid plaque, the number of arterial segments, and ABI). In all analyses, a one standard deviation decrease in the continuous exposure variables (BUA 13.7 and $14.7 \mathrm{~dB} / \mathrm{MHz}$; SOS 37.5 and $33.5 \mathrm{~m} / \mathrm{s}$; and stiffness index 18.1 and 17.5 in men and women, resp.) was modelled. In models with the categorical exposure variable, osteoporotic fracture risk, a low osteoporotic fracture risk was used as a reference category. The outcomes IMT and ABI entered the regression models as continuous variables, while carotid plaque was dichotomized (present/not present). Finally, the number of arterial segments affected by plaque was used as a continuous variable. It was transformed (log (number of segments +1$)$ ) before being entered in the ANOVA or linear regression models. We report adjusted means with $95 \%$ confidence intervals from the ANOVA, $\beta$-coefficients with standard errors and $p$ values from the linear regression models and odds ratios with $95 \%$ confidence intervals from the logistic regression models. Results from unadjusted and fully adjusted models, including age, BMI, smoking status, physical inactivity, risky alcohol consumption, diabetes mellitus, dyslipidemia, hypertension, and, in women, intake of estrogens (oral contraceptives or hormone replacement therapy) and years since menopause, are presented.

Among the SHIP-2 and SHIP-Trend participants, a subpopulation underwent the ABI examination. To account for the possible selection bias according to nonparticipation, 
inverse probability weights using sex, age, smoking, education, equivalence household income, blood pressure, antihypertensive medication, diabetes mellitus, HbAlc, lipids, and BMI as explanatory variables were applied [20]. All statistical analyses were performed with SAS 9.4 (SAS Institute Inc., Cary, North Carolina, USA).

\section{Results}

The majority of men (68.2\%) and women (61.2\%) in our study population had a low QUS-based osteoporotic fracture risk. On the other side, $4.4 \%$ of men and $6.5 \%$ of women had a high and $27.4 \%$ and $32.3 \%$ a medium QUS-based osteoporotic fracture risk, respectively. The presence of cardiovascular risk factors differed between the three fracture risk groups. Men with a high fracture risk were older, had lower BMI, were more often smokers or physically inactive, and were more often risky alcohol consumers than men with a low or medium fracture risk. Women with a high risk were also older than those with a low or medium risk, had more often diabetes, dyslipidemia, and hypertension but did not differ with respect to BMI, physical inactivity, and risky alcohol consumption. Besides, the CCA-IMT, the presence of plaques, and the number of arterial segments affected by plaque significantly increased over the three risk groups in men and women (Table 1).

In unadjusted linear regression analyses, inverse associations of BUA, SOS, stiffness index, or the QUS-based osteoporotic fracture risk with the CCA-IMT were found. In men, for example, a decrease in the stiffness index by 18.1 points was associated with an increase in CCA-IMT of $0.016 \mathrm{~cm}$. After adjustment for age (data not shown) and also in fully adjusted models, however, the associations were not confirmed (Table 2).

Regarding plaques, unadjusted logistic regression analyses demonstrated significantly increased odds for the presence of plaques with decreasing QUS-based parameters in both sexes. In fully adjusted models, these associations were confirmed in men but not in women (Table 3). Men with a high fracture risk had significantly increased odds for plaque occurrence compared to men with a low risk. In men, also, the number of arterial segments affected by plaque significantly increased over the risk categories (Figure 1) in unadjusted as well as in the fully adjusted ANOVA. In women, similar findings were made in unadjusted analyses, whereas in fully adjusted models, the results disappeared.

In the subpopulation with ABI measurement, the proportions of men and women with high, medium, or low fracture risk were comparable to the proportions in the whole population (men and women high risk: $69.2 \%$ and $62.1 \%$; medium risk: $26.3 \%$ and $32.0 \%$; and low risk: $4.5 \%$ and $5.9 \%$; for more details, see Supplemental Table 1). The ABI was similar between the three risk groups [median (1st-3rd quartiles) for men and women with a high risk: $1.12(1.07-1.19)$ and 1.11 (1.07-1.18), medium risk: $1.13(1.07-1.19)$ and 1.13 (1.08-1.19), and low risk: $1.12(1.04-1.21)$ and 1.13 (1.081.18), resp.]. Fully adjusted, sex-specific linear regression models (Supplemental Table 2) revealed no significant association between the QUS parameters and the ABI in men or women.

\section{Discussion}

In the present study, we demonstrated associations between decreased bone quality, defined by heel QUS parameters, and the presence of atherosclerotic carotid artery plaque in men. These associations were independent of major cardiovascular risk factors, including dyslipidemia, diabetes, and hypertension. In contrast, our data do not provide evidence for relevant independent associations of heel bone quality with CCA-IMT or ABI.

Traditionally, osteoporosis and atherosclerosis have been regarded as independent processes sharing common risk factors, for example, aging [21]. Recent evidence from cell culture as well as epidemiological studies, however, points to an age-independent association between osteoporosis and atherosclerosis [6, 22]. Defects in bone mineralization and arterial calcification were attributed of having a similar pathogenesis [6], and associations between bone quality and atherosclerotic changes independent of cardiovascular risk factors have been proposed.

Following this hypothesis, the previous studies $[23,24]$ demonstrated associations between decreased BMD and increased IMT in elderly individuals. For example, the San Antonio Family Osteoporosis Study demonstrated that decreased BMD at various sites is correlated with carotid artery IMT in older women and men [23]. Among the SHIP-2 and SHIP-Trend participants, highly significant inverse associations between the QUS-based parameters and the carotid artery IMT were observed in unadjusted models but turned nonsignificant after adjustment for cardiovascular risk factors. Comparable observations were made by Frost et al. [25], who found associations between spine BMD and IMT, but the relationship was not significant after adjustment for age, mean arterial pressure, and triglycerides.

While our data thus argues against independent associations between bone metabolism and IMT, it provides evidence for a male-specific relation with carotid artery plaques. CCA-IMT and plaques are biologically and genetically distinct markers of atherosclerosis. They differ with respect to specific pattern of risk factors, their pathogenesis, and their ability to predict cardiovascular and cerebrovascular events [26]. Numerous factors including inflammation, protein metabolism, and oxidative stress were proposed to promote development of disease in both osteoporotic and atherosclerotic changes [7]. These factors are likely involved in the pathogenesis of focal atherosclerotic plaques but are less important for an arterial wall thickening of the CCA, which may explain the strong association between QUS-based bone quality and plaque occurrence and the nonsignificant association between bone quality and IMT. Corresponding observations of associations between bone quality and the presence of plaques were reported from various studies $[5,25,27,28]$. For example, the Troms $\varnothing$ study [5] showed that BMD was associated with calcified echogenic plaque independent of potential confounders, mediators, and shared risk factors in men and women [5]. Also, Hyder et al. 


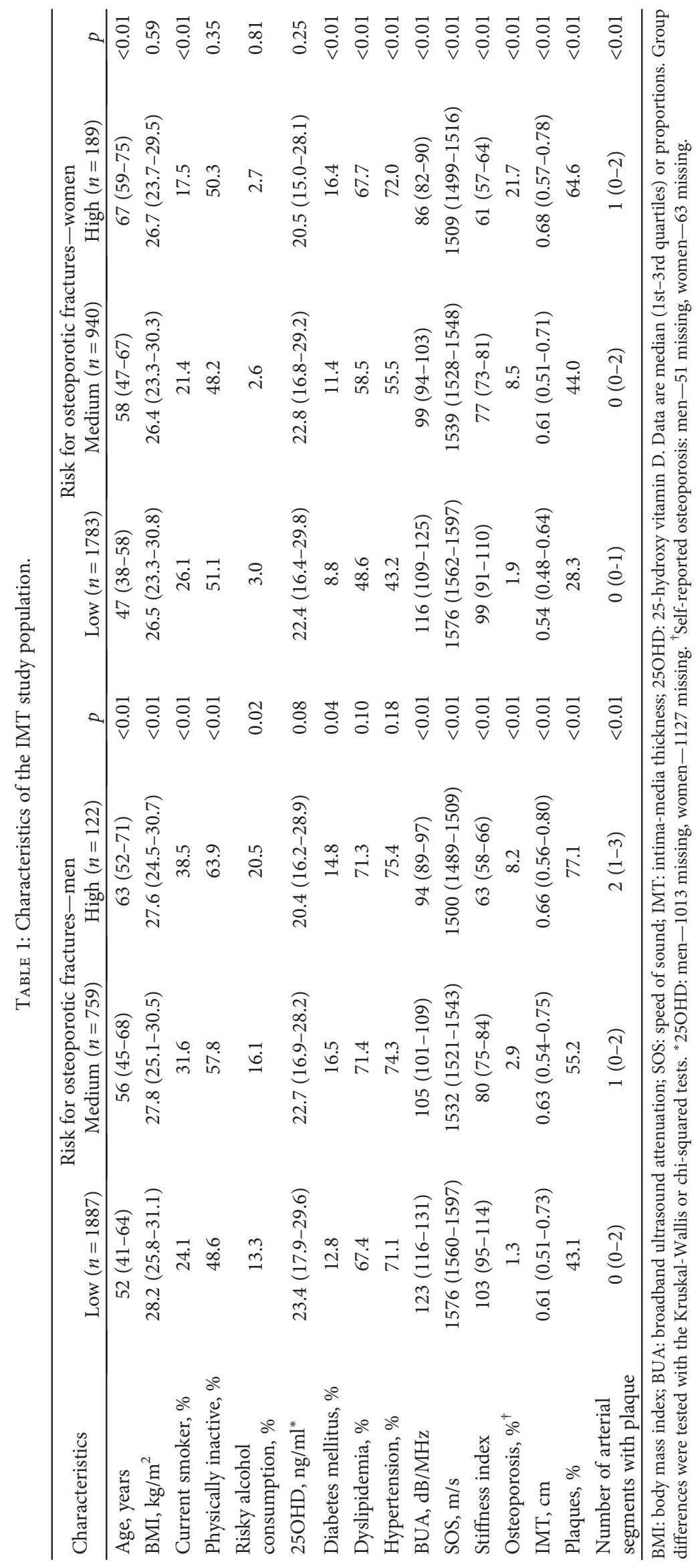


TABLE 2: Associations between a decrease in QUS-based parameters and IMT.

\begin{tabular}{|c|c|c|c|c|c|c|c|}
\hline \multirow{2}{*}{ Exposure } & \multirow{2}{*}{ Adjustment } & \multicolumn{3}{|c|}{ Men } & \multicolumn{3}{|c|}{ Women } \\
\hline & & $\beta$-Coefficient & SE & $p$ & $\beta$-Coefficient & SE & $p$ \\
\hline BUA & & 0.009 & 0.003 & $<0.01$ & 0.034 & 0.003 & $<0.01$ \\
\hline SOS & & 0.020 & 0.003 & $<0.01$ & 0.038 & 0.002 & $<0.01$ \\
\hline Stiffness index & Unadjusted & 0.016 & 0.003 & $<0.01$ & 0.039 & 0.002 & $<0.01$ \\
\hline Risk: medium versus low & & 0.022 & 0.007 & $<0.01$ & 0.053 & 0.005 & $<0.01$ \\
\hline Risk: high versus low & & 0.052 & 0.148 & $<0.01$ & 0.113 & 0.010 & $<0.01$ \\
\hline BUA & & -0.002 & 0.002 & 0.32 & -0.003 & 0.002 & 0.13 \\
\hline SOS & & -0.003 & 0.002 & 0.28 & -0.000 & 0.002 & 0.87 \\
\hline Stiffness index & Fully adjusted & -0.003 & 0.002 & 0.25 & -0.002 & 0.002 & 0.34 \\
\hline Risk: medium versus low & & -0.004 & 0.004 & 0.34 & -0.003 & 0.004 & 0.50 \\
\hline Risk: high versus low & & -0.010 & 0.008 & 0.19 & -0.007 & 0.008 & 0.38 \\
\hline
\end{tabular}

BUA: broadband ultrasound attenuation; IMT: intima-media thickness; QUS: quantitative ultrasound; SD: standard deviation; SE: standard error; SOS: speed of sound. $\beta$-Coefficients, standard errors (SE), and $p$ values from linear regression models. For BUA, SOS, and stiffness index, a one standard deviation decrease was modelled. A one standard deviation of BUA for men and women: 13.7 and $14.7 \mathrm{~dB} / \mathrm{MHz}$; SOS: 37.5 and $33.5 \mathrm{~m} / \mathrm{s}$; stiffness index: 18.1 and 17.5 . Full adjustment for age, body mass index, smoking status, physical inactivity, risky alcohol consumption, diabetes mellitus, dyslipidemia, hypertension, and, in women, additionally intake of estrogens (oral contraceptives or hormone replacement therapy) and years since menopause.

TABle 3: Associations between a decrease in QUS-based parameters and plaques.

\begin{tabular}{lcrr}
\hline Exposure & Adjustment & \multicolumn{2}{c}{ Odds ratio (95\% confidence interval) } \\
Women & $1.68(1.54-1.82)$ \\
\hline BUA & & $1.32(1.22-1.42)$ & $1.74(1.59-1.89)$ \\
SOS & Unadjusted & $1.46(1.35-1.59)$ & $1.80(1.65-1.96)$ \\
Stiffness index & & $1.43(1.33-1.55)$ & $1.99(1.69-2.35)$ \\
Risk: medium versus low & & $1.62(1.37-1.93)$ & $4.61(3.36-6.32)$ \\
Risk: high versus low & & $4.43(2.87-6.81)$ & $0.98(0.88-1.10)$ \\
BUA & Fully adjusted & $1.23(1.11-1.37)$ & $1.01(0.90-1.12)$ \\
SOS & & $1.20(1.08-1.34)$ & $1.00(0.90-1.11)$ \\
Stiffness index & & $1.24(1.11-1.38)$ & $0.90(0.73-1.20)$ \\
Risk: medium versus low & $1.24(0.99-1.55)$ & $0.93(0.63-1.38)$ \\
Risk: high versus low & & $2.93(1.70-5.06)$ & \\
\hline
\end{tabular}

BUA: broadband ultrasound attenuation; QUS: quantitative ultrasound; SOS: speed of sound. Odds ratios and 95\% confidence intervals from logistic regression models. For BUA, SOS, and stiffness index, a one standard deviation decrease was modelled. One standard deviation of BUA for men and women: 13.7 and $14.7 \mathrm{~dB} / \mathrm{MHz}$; SOS: 37.5 and $33.5 \mathrm{~m} / \mathrm{s}$; stiffness index: 18.1 and 17.5. Full adjustment for age, body mass index, smoking status, physical inactivity, risky alcohol consumption, diabetes mellitus, dyslipidemia, hypertension, and, in women, additionally intake of estrogens (oral contraceptives or hormone replacement therapy) and years since menopause.

investigated in 904 postmenopausal women and 929 men an independent association between echogenically measured carotid plaque and lower volumetric trabecular lumbar BMD [7]. While our results were restricted to men, other studies reported associations between carotid plaques and BMD only in women $[10,27]$ or were restricted to women $[25,29,30]$. Nevertheless, also, male-specific associations between $\mathrm{BMD}$ and plaques [7, 8, 31, 32] were previously reported.

The sex differences in the examined associations may be explained by differences in bone metabolism between men and women. Men have a higher peak of bone mass than women [33], and osteoporosis emerges later in life and is more often due to secondary causes [34]. At the same time, osteoporosis is strongly associated with comorbidities and frailty in men than in women [35] and men have a higher fracture-related mortality than women [36]. Thus, men presenting with clinical signs of osteoporosis may be generally more frail and present with a larger number of comorbidities than men without such signs. This probably explains the increased risk of cardiovascular pathologies with decreasing bone quality, independent of age, and further cardiovascular risk factors. In these men, heightened awareness regarding atherosclerotic changes may be of clinical importance.

In previous studies, bone quality was rarely assessed by QUS, instead dual-X-ray-absorptiometry (DXA) was performed. DXA provides information on BMD and is the recommended measurement in the diagnosis and monitoring of osteoporosis [37, 38]. However, also, QUS provides reliable results to predict fracture risk $[39,40]$ and has the advantage of being radiation-free and easy to handle. To our knowledge, there are only few studies using QUS to 


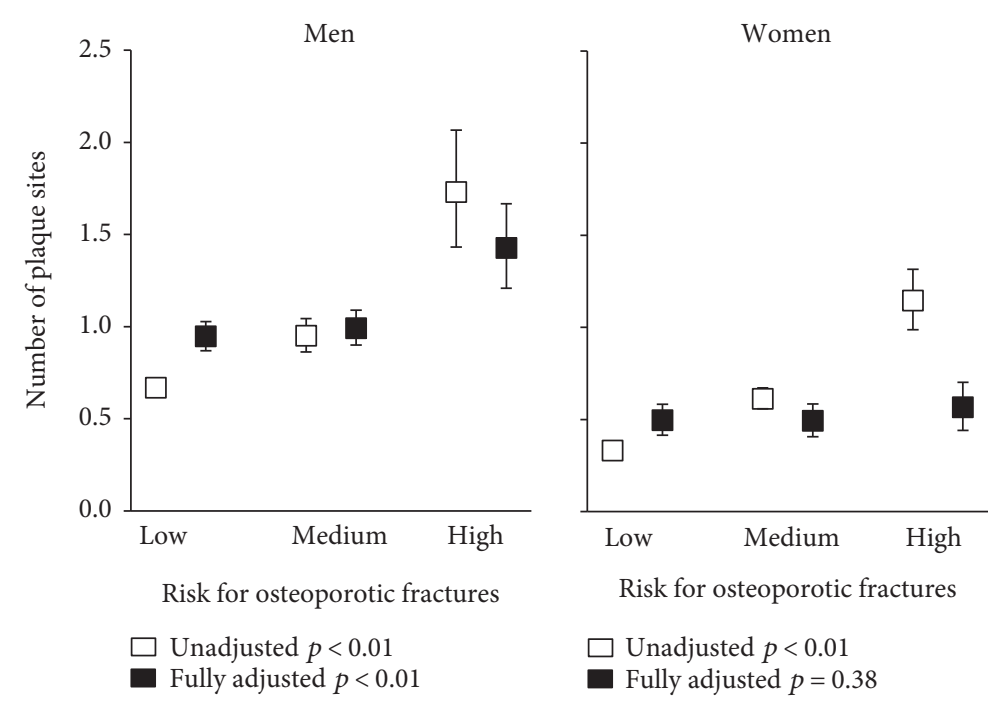

FIGURE 1: Adjusted mean number of plaque sites according to QUS-based osteoporotic fracture risk by sex. ANOVA was adjusted for age, body mass index, smoking status, physical inactivity, risky alcohol consumption, diabetes mellitus, dyslipidemia, hypertension, and, in women, additionally intake of estrogens (oral contraceptives or hormone replacement therapy) and years since menopause. The number of arterial segments affected by plaque was transformed (log (number of plaque sites +1$)$ ) before being entered in the model and back-transformed for display in the figure.

investigate the association between bone quality and atherosclerosis [13, 41-43]. These studies reported conflicting results, but predominantly suggest an association between QUS and atherosclerotic changes, which is in line with our results.

Next to IMT and plaques, we assessed the relation between bone stiffness and ABI, as a marker of vascular calcification and increased vessel stiffness [44]. It is known that decreased blood flow to the lower limbs caused by PAD leads to compromising the bone quality [45]. In line with this, the Rotterdam Study [46], including 5268 individuals, reported a significantly increased risk for PAD in women with a low femoral neck BMD even after adjusting for age. Our study failed to show an association between bone stiffness and $\mathrm{ABI}$. Yet, $\mathrm{PAD}$ as defined by $\mathrm{ABI} \leq 0.9$ was rare in our study population; only $1.53 \%$ of participants entailed these values, and early stages of PAD may not impair bone health [12]. This may have prevented us from detecting a respective association. Moreover, our results are in line with the majority of previous studies that report no $[43,47]$ or only weak $[12,48]$ associations between BMD and arterial stiffness.

Our study has several strengths and limitations. Strengths result from the large sample including men and women over a large age range (20-93 years). Further, all study participants underwent intensive medical examinations with highly standardized procedures, assuring high data quality. Moreover, we adjusted our models for interfering covariates to assess the impact of comorbidities.

Besides these strengths, our study has its limitations. First, the cross-sectional design does not allow assessing causality between the measures. Second, cardioprotective drugs were taken by a large proportion $(40.7 \%)$ of our study population. The intake of such medication reduces the cardiovascular risk and may lead to an underestimation of the effect of the examined associations. Third, BMD measurements were not available; thus, our study is not directly comparable to other studies using BMD. However, the QUS-based results provide complementary evidence and, in a populationbased research setting, offer the advantage of being simpler, less expensive, and free of ionizing radiation. Fourth, ABI measurements were only available in a subsample. To rule out that nonparticipation resulted in a selection bias, we weighted the respective data based on social-demographic and health-related variables. Fifth, 25OHD concentrations were unavailable in nearly $40 \%$ of the study population, and vitamin D intake was reported by only 29 subjects. Therefore, we refrained from including that information in the analyses. Sixth, our study was performed exclusively in Caucasian European subjects; thus, our results may not be directly transferrable to other regions or ethnicities.

In conclusion, our data argue against an independent role of bone metabolism in atherosclerotic changes in women. Yet, in men, associations with atherosclerotic changes, especially formation of plaques, seem present. Thus, men presenting with clinical signs of osteoporosis may be at increased risk for atherosclerotic disease. Further studies are needed to understand the relation between calcified plaque and decreased bone quality.

\section{Conflicts of Interest}

The authors declare that there is no conflict of interest that could be perceived as prejudicing the impartiality of the research reported.

\section{Acknowledgments}

SHIP is part of the Community Medicine Research net of the University of Greifswald, Germany, which is funded by the Federal Ministry of Education and Research (Grant nos. 
01ZZ9603, 01ZZ0103, and 01ZZ0403), the Ministry of Cultural Affairs, and the Social Ministry of the Federal State of Mecklenburg-West Pomerania. This work is also part of the research project Greifswald Approach to Individualized Medicine (GANI_MED), which is funded by the Federal Ministry of Education and Research and the Ministry of Cultural Affairs of the Federal State of Mecklenburg-West Pomerania (03IS2061A). Dr. A. Hannemann is supported by a Gollwitz-Meier scholarship.

\section{References}

[1] E. Hernlund, A. Svedbom, M. Ivergard et al., "Osteoporosis in the European Union: medical management, epidemiology and economic burden. A report prepared in collaboration with the International Osteoporosis Foundation (IOF) and the European Federation of Pharmaceutical Industry Associations (EFPIA)," Archives of Osteoporosis, vol. 8, p. 136, 2013.

[2] T. M. Doherty, L. A. Fitzpatrick, D. Inoue et al., "Molecular, endocrine, and genetic mechanisms of arterial calcification," Endocrine Reviews, vol. 25, pp. 629-672, 2004.

[3] S. Allender, P. Scarborough, and V. Peto, "European cardiovascular disease statistics: 2008 edition, European Heart Network," April 2017, https://www.bhforguk/publications/ statistics/european-cardiovascular-disease-statistics-2008.

[4] World Health Organization, "Cardiovascular diseases (CVDs): key facts," March 2017, http://www.whoint/mediacentre/ factsheets/fs317/en/.

[5] L. Jorgensen, O. Joakimsen, G. K. Rosvold Berntsen, I. Heuch, and B. K. Jacobsen, "Low bone mineral density is related to echogenic carotid artery plaques: a population-based study," American Journal of Epidemiology, vol. 160, pp. 549-556, 2004.

[6] T. M. Doherty, K. Asotra, L. A. Fitzpatrick et al., "Calcification in atherosclerosis: bone biology and chronic inflammation at the arterial crossroads," Proceedings of the National Academy of Sciences of the United States of America, vol. 100, pp. 11201-11206, 2003.

[7] J. A. Hyder, M. A. Allison, E. Barrett-Connor et al., "Bone mineral density and atherosclerosis: the multi-ethnic study of atherosclerosis, abdominal aortic calcium study," Atherosclerosis, vol. 209, pp. 283-289, 2010.

[8] H. T. Lee, J. Shin, S. Y. Min et al., "Relationship between bone mineral density and a 10-year risk for coronary artery disease in a healthy Korean population: the Korea National Health and Nutrition Examination Survey 2008-2010," Coronary Artery Disease, vol. 26, pp. 66-71, 2015.

[9] N. L. Kim, H. M. Jang, S. K. Kim, K. D. Ko, I. C. Hwang, and H. S. Suh, "Association of arterial stiffness and osteoporosis in healthy men undergoing screening medical examination," Journal of Bone Metabolism, vol. 21, pp. 133-141, 2014.

[10] N. Campos-Obando, M. Kavousi, J. E. Roeters van Lennep et al., "Bone health and coronary artery calcification: the Rotterdam study," Atherosclerosis, vol. 241, pp. 278-283, 2015.

[11] D. K. Liang, X. J. Bai, B. Wu et al., "Associations between bone mineral density and subclinical atherosclerosis: a crosssectional study of a Chinese population," The Journal of Clinical Endocrinology and Metabolism, vol. 99, pp. 469-477, 2014.

[12] D. von Muhlen, M. Allison, S. K. Jassal, and E. Barrett-Connor, "Peripheral arterial disease and osteoporosis in older adults: the Rancho Bernardo study," Osteoporosis International, vol. 20, pp. 2071-2078, 2009.
[13] K. Hirose, H. Tomiyama, R. Okazaki et al., "Increased pulse wave velocity associated with reduced calcaneal quantitative osteo-sono index: possible relationship between atherosclerosis and osteopenia," The Journal of Clinical Endocrinology and Metabolism, vol. 88, pp. 2573-2578, 2003.

[14] P. Pennisi, S. S. Signorelli, S. Riccobene et al., "Low bone density and abnormal bone turnover in patients with atherosclerosis of peripheral vessels," Osteoporosis International, vol. 15, pp. 389-395, 2004.

[15] U. John, B. Greiner, E. Hensel et al., "Study of health in Pomerania (SHIP): a health examination survey in an east German region: objectives and design," Sozial- und Präventivmedizin, vol. 46, pp. 186-194, 2001

[16] H. Volzke, D. Alte, C. O. Schmidt et al., "Cohort profile: the study of health in Pomerania," International Journal of Epidemiology, vol. 40, pp. 294-307, 2011.

[17] R. M. Berg, H. Wallaschofski, M. Nauck et al., "Positive association between adipose tissue and bone stiffness," Calcified Tissue International, vol. 97, pp. 40-49, 2015.

[18] V. Aboyans, M. H. Criqui, P. Abraham et al., "Measurement and interpretation of the ankle-brachial index: a scientific statement from the American Heart Association," Circulation, vol. 126, pp. 2890-2909, 2012.

[19] A. S. Levey, T. Greene, J. W. Kusek, and G. J. Beck, “A simplified equation to predict glomerular filtration rate from serum creatinine," Journals of the American Society of Nephrology, vol. 11, 2000, Abstract A0828.

[20] L. Li, C. Shen, X. Li, and J. M. Robins, "On weighting approaches for missing data," Statistical Methods in Medical Research, vol. 22, pp. 14-30, 2013.

[21] D. Hamerman, "Osteoporosis and atherosclerosis: biological linkages and the emergence of dual-purpose therapies," QJM: Monthly Journal of the Association of Physicians, vol. 98, pp. 467-484, 2005.

[22] P. Anagnostis, A. Karagiannis, A. I. Kakafika, K. Tziomalos, V. G. Athyros, and D. P. Mikhailidis, "Atherosclerosis and osteoporosis: age-dependent degenerative processes or related entities?," Osteoporosis International, vol. 20, pp. 197-207, 2009.

[23] J. R. Shaffer, C. M. Kammerer, D. L. Rainwater et al., "Decreased bone mineral density is correlated with increased subclinical atherosclerosis in older, but not younger, Mexican American women and men: the San Antonio family osteoporosis study," Calcified Tissue International, vol. 81, pp. 430441, 2007.

[24] J. Tamaki, M. Iki, Y. Hirano et al., "Low bone mass is associated with carotid atherosclerosis in postmenopausal women: the Japanese population-based osteoporosis (JPOS) cohort study," Osteoporosis International, vol. 20, pp. 53-60, 2009.

[25] M. L. Frost, R. Grella, S. C. Millasseau et al., "Relationship of calcification of atherosclerotic plaque and arterial stiffness to bone mineral density and osteoprotegerin in postmenopausal women referred for osteoporosis screening," Calcified Tissue International, vol. 83, pp. 112-120, 2008.

[26] P. J. Touboul, M. G. Hennerici, S. Meairs et al., "Mannheim carotid intima-media thickness and plaque consensus (2004-2006-2011). An update on behalf of the advisory board of the $3 \mathrm{rd}$, 4th and 5th watching the risk symposia, at the 13th, 15th and 20th European Stroke Conferences, Mannheim, Germany, 2004, Brussels, Belgium, 2006, and Hamburg, Germany, 2011," Cerebrovascular Diseases, vol. 34, pp. 290-296, 2012. 
[27] S. N. Kim, H. S. Lee, H. S. Nam et al., "Carotid intima-media thickness is inversely related to bone density in female but not in male patients with acute stroke," Journal of Neuroimaging, vol. 26, pp. 83-88, 2016.

[28] O. Uyama, Y. Yoshimoto, Y. Yamamoto, and A. Kawai, "Bone changes and carotid atherosclerosis in postmenopausal women," Stroke, vol. 28, pp. 1730-1732, 1997.

[29] H. Sumino, S. Ichikawa, S. Kasama et al., "Elevated arterial stiffness in postmenopausal women with osteoporosis," Maturitas, vol. 55, pp. 212-218, 2006.

[30] M. Varri, T. P. Tuomainen, R. Honkanen et al., "Carotid intima-media thickness and calcification in relation to bone mineral density in postmenopausal women-the OSTPREBBA study," Maturitas, vol. 78, pp. 304-309, 2014.

[31] J. J. Carr, T. C. Register, F. C. Hsu et al., "Calcified atherosclerotic plaque and bone mineral density in type 2 diabetes: the diabetes heart study," Bone, vol. 42, pp. 43-52, 2008.

[32] J. H. Magnus and D. L. Broussard, "Relationship between bone mineral density and myocardial infarction in US adults," Osteoporosis International, vol. 16, pp. 2053-2062, 2005.

[33] D. A. Bailey, A. D. Martin, H. A. McKay, S. Whiting, and R. Mirwald, "Calcium accretion in girls and boys during puberty: a longitudinal analysis," Journal of Bone and Mineral Research, vol. 15, pp. 2245-2250, 2000.

[34] S. P. Tuck and H. K. Datta, "Osteoporosis in the aging male: treatment options," Clinical Interventions in Aging, vol. 2, pp. 521-536, 2007.

[35] M. Laurent, E. Gielen, F. Claessens, S. Boonen, and D. Vanderschueren, "Osteoporosis in older men: recent advances in pathophysiology and treatment," Best Practice \& Research. Clinical Endocrinology \& Metabolism, vol. 27, pp. 527-539, 2013.

[36] J. R. Center, T. V. Nguyen, D. Schneider, P. N. Sambrook, and J. A. Eisman, "Mortality after all major types of osteoporotic fracture in men and women: an observational study," Lancet, vol. 353, pp. 878-882, 1999.

[37] J. A. Kanis, E. V. McCloskey, H. Johansson et al., "European guidance for the diagnosis and management of osteoporosis in postmenopausal women," Osteoporosis International, vol. 24, pp. 23-57, 2013.

[38] Y. Lu, H. K. Genant, J. Shepherd et al., "Classification of osteoporosis based on bone mineral densities," Journal of Bone and Mineral Research, vol. 16, pp. 901-910, 2001.

[39] Dachverband Osteologie e.V. Prophylaxe, Diagnostik und Therapie der Osteoporose bei Männern ab dem 60 . Lebensjahr und bei postmenopausalen Frauen, "S3-Leitlinie des Dachverbands der Deutschsprachigen Wissenschaftlichen Osteologischen Gesellschaften (DVO) e.V. 2014," April 2017, http://www.dv-osteologie.org/dvo_leitlinien/osteoporoseleitlinie-2014.

[40] J. Shams, A. B. Spitzer, A. M. Kennelly, and L. L. Tosi, "Bone quality: educational tools for patients, physicians, and educators," Clinical Orthopaedics and Related Research, vol. 469, pp. 2248-2259, 2011.

[41] S. Yamada, M. Inaba, H. Goto et al., "Significance of intimamedia thickness in femoral artery in the determination of calcaneus osteo-sono index but not of lumbar spine bone mass in healthy Japanese people," Osteoporosis International, vol. 16, pp. 64-70, 2005.

[42] M. M. Pinheiro, C. M. Castro, and V. L. Szejnfeld, "Low femoral bone mineral density and quantitative ultrasound are risk factors for new osteoporotic fracture and total and cardiovascular mortality: a 5-year population-based study of Brazilian elderly women," The Journals of Gerontology. Series A, Biological Sciences and Medical Sciences, vol. 61, pp. 196203, 2006

[43] S. C. van Dijk, R. T. de Jongh, A. W. Enneman et al., "Arterial stiffness is not associated with bone parameters in an elderly hyperhomocysteinemic population," Journal of Bone and Mineral Metabolism, vol. 34, pp. 99-108, 2016.

[44] K. I. Paraskevas, I. Kotsikoris, S. A. Koupidis, A. D. Giannoukas, and D. P. Mikhailidis, "Ankle-brachial index: a marker of both peripheral arterial disease and systemic atherosclerosis as well as a predictor of vascular events," Angiology, vol. 61, pp. 521-523, 2010.

[45] M. Laroche, L. Moulinier, P. Leger, D. Lefebvre, B. Mazieres, and $\mathrm{H}$. Boccalon, "Bone mineral decrease in the leg with unilateral chronic occlusive arterial disease," Clinical and Experimental Rheumatology, vol. 21, pp. 103-106, 2003.

[46] M. van der Klift, H. A. Pols, A. E. Hak, J. C. Witteman, A. Hofman, and C. E. de Laet, "Bone mineral density and the risk of peripheral arterial disease: the Rotterdam study," Calcified Tissue International, vol. 70, pp. 443-449, 2002.

[47] Y. Q. Wang, P. T. Yang, H. Yuan et al., "Low bone mineral density is associated with increased arterial stiffness in participants of a health records based study," Journal of Thoracic Disease, vol. 7, pp. 790-798, 2015.

[48] S. Y. Wong, T. Kwok, J. Woo et al., "Bone mineral density and the risk of peripheral arterial disease in men and women: results from Mr. and Ms Os, Hong Kong," Osteoporosis International, vol. 16, pp. 1933-1938, 2005. 


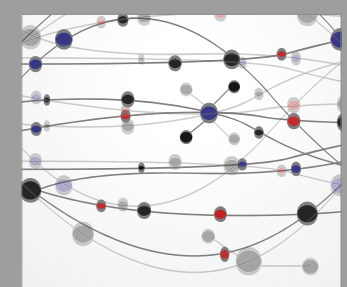

The Scientific World Journal
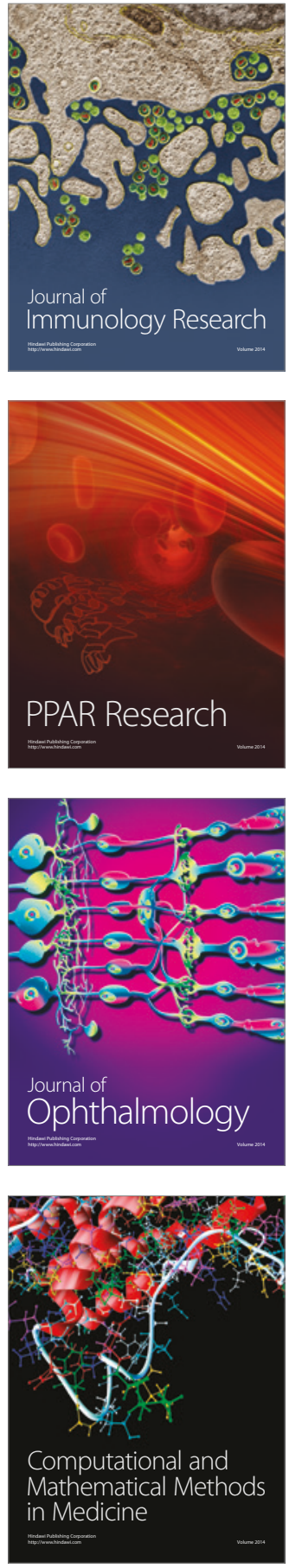

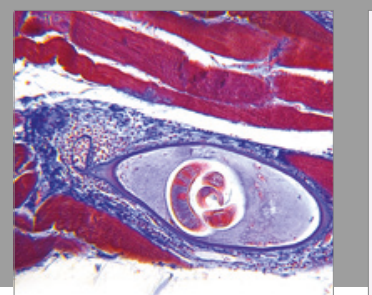

Gastroenterology Research and Practice
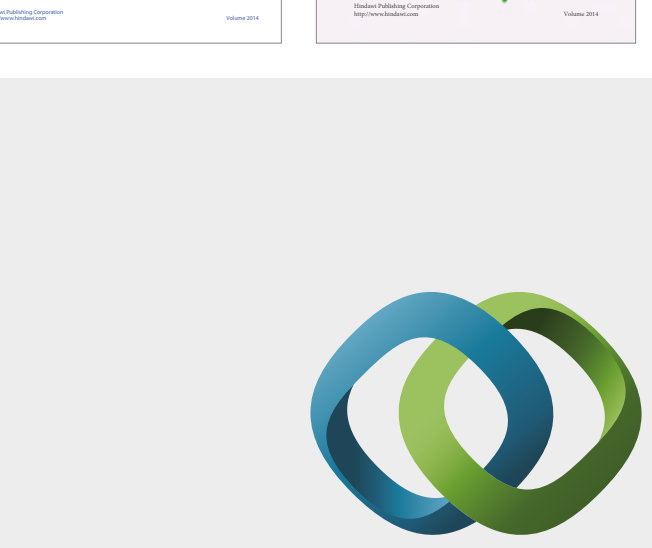

\section{Hindawi}

Submit your manuscripts at

https://www.hindawi.com
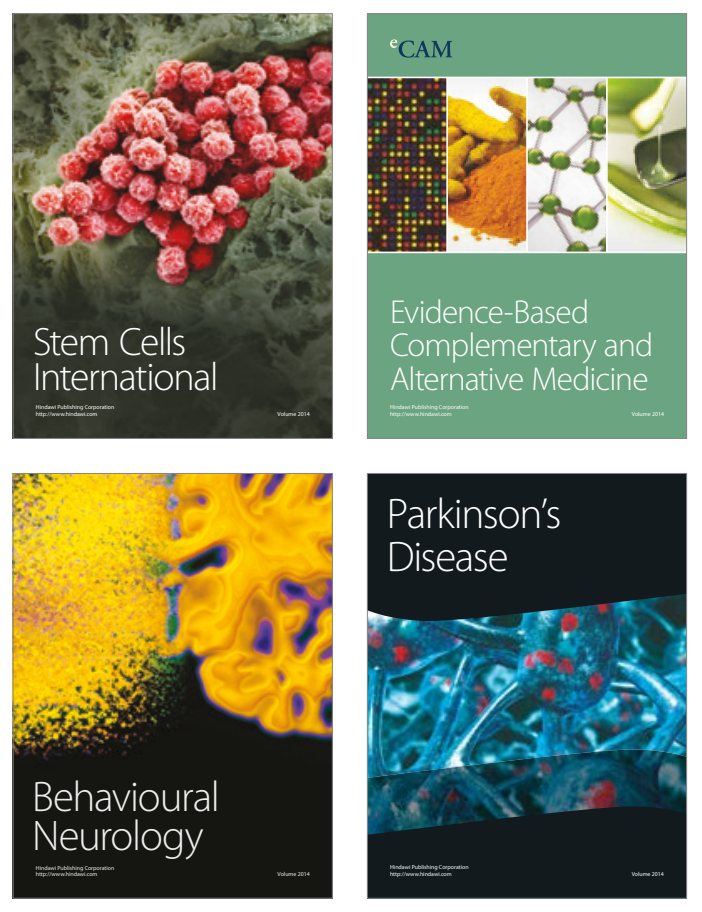
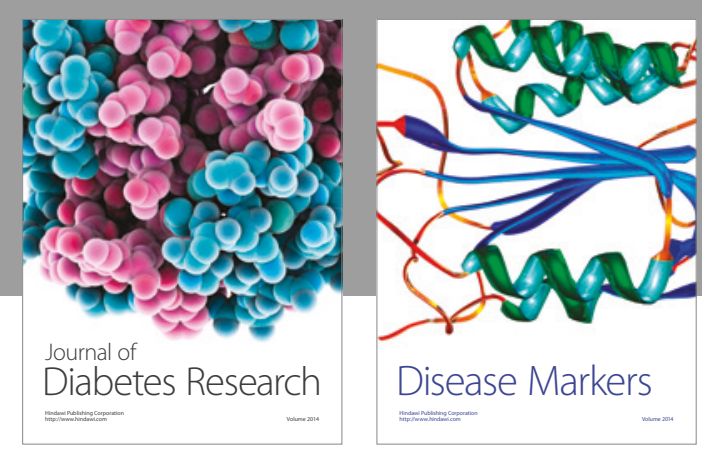

Disease Markers
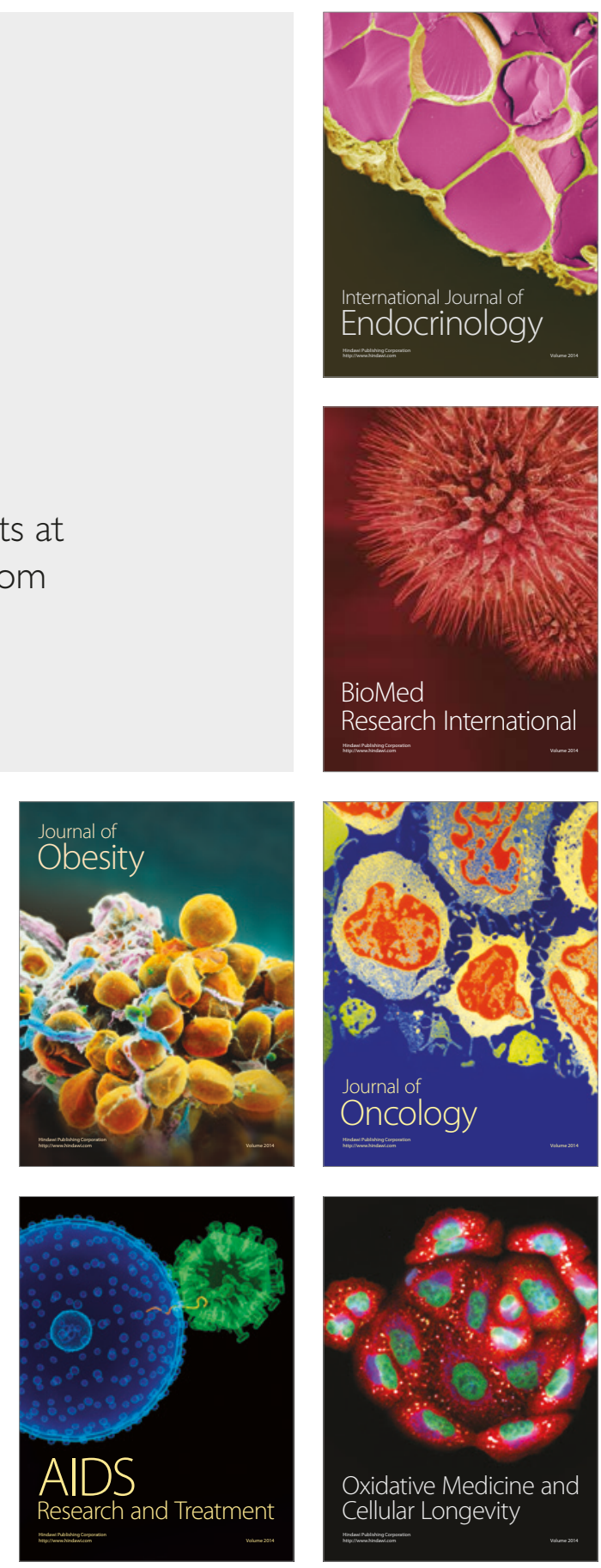